\title{
Anesthetic and Pharmaco-economic Outcomes in Prostate Brachytherapy
}

AUTHORS: Anita Sarmah MD FRCA, ${ }^{*}$ E. Alexander Williams MD FRCPC, ${ }^{\dagger}$ Jenny LamMcCulloch MSc, Joseph Kay MD FRCPC

\author{
AFFILIATIONS: \\ *Department of Anesthesia, Sunnybrook and Women's College Health Sciences Centre, University \\ of Toronto, 2075 Bayview Avenue, Toronto, Ontario M4N 3M5 \\ ${ }^{\dagger}$ Department of Anesthesia, Belleville General Hospital, 265 Dundas Street East, \\ Belleville, Ontario K8N 5A9
}

INTRODUCTION: This randomized controlled trial was conducted to compare outcome and costeffectiveness of general anesthetic (GA) versus low dose lidocaine saddle block (SB), in patients undergoing ambulatory transperineal prostate $\mathrm{I}^{125}$ seed implantation.

METHODS: After IRB approval, 119 consented patients were randomized to receive either a standardized GA or low dose SB. The GA group received intravenous fentanyl $0.5-1 \mu \mathrm{gkg}^{-1}$, propofol $1-4 \mathrm{mgkg}^{-1}$, and maintenance with sevoflurane and nitrous oxide in oxygen. SB patients received intravenous midazolam $1-3 \mathrm{mg}$ and an intrathecal injection of $2 \%$ lidocaine $1 \mathrm{~mL}, 10 \%$ dextrose $1 \mathrm{~mL}$ and fentanyl $10 \mu \mathrm{g}$, total volume $2.2 \mathrm{ml} .{ }^{1}$ After 5 minutes of sitting, patients were placed in $15^{\circ}$ reverse Trendelenberg lithotomy position. Intraoperative hypotension, postoperative VAS, and time to ambulation and discharge were recorded. Patients were contacted three days postdischarge to assess satisfaction, analgesic use and side effects including Transient Neurologic Symptoms (TNS). All costs including Direct and Natural units ${ }^{2}$ were calculated in Canadian Dollars. Treatment differences between binary outcomes such as side effects were calculated using two-sided $\chi^{2}$ test. Differences in time between groups were calculated using a t-test.

RESULTS: Each group consisted of 59 patients (one withdrawal due to GERD). In the PACU, SB patients had consistently lower VAS scores than GA patients and were faster to ambulate and discharge. Intraoperative hypotension and use of postoperative analgesics was exclusive to the GA group (24 and 16 patients, respectively). No TNS was reported. All patients were extremely satisfied with their anesthetic experience. The cost for the GA group was $\$ 87.45 \pm \$ 24.77$, with the SB group significantly lower at $\$ 79.97 \pm \$ 14.26(\mathrm{p} \leq 0.05)$.

\begin{tabular}{|l|c|c|c|}
\hline & Time to Ambulation (min)* & Time to Discharge (min)* & $\begin{array}{c}\text { Cost per patient } \\
(\text { \$CDN)* }\end{array}$ \\
\hline SB & $105.53 \pm 26.38$ & $108.67 \pm 23.25$ & $79.97 \pm 14.26$ \\
\hline GA & $121.71 \pm 43.73$ & $124 \pm 37.28$ & $87.45 \pm 24.77$ \\
\hline
\end{tabular}

Values expressed as mean \pm standard deviation; ${ }^{*} \mathrm{p} \leq 0.05$

DISCUSSION: Low dose SB provides superior anesthesia and analgesia with minimal side effects, shorter length of hospital stay and increased cost effectiveness.

REFERENCES: 1. IARS Congress, Hawaii, 2000; 2. Anesthesia 1998;53:782-88. 